\title{
8-2018
}

\section{Validation of the Registered Nurse Assessment of Readiness for Hospital Discharge Scale}

\author{
Kathleen L. Bobay \\ Loyola University Chicago, kbobay@luc.edu \\ Marianne E. Weiss \\ Debra Oswald \\ Olga Yakusheva
}

Follow this and additional works at: https://ecommons.luc.edu/nursing_facpubs

Part of the Critical Care Nursing Commons

\section{Recommended Citation}

Bobay, Kathleen L.; Weiss, Marianne E.; Oswald, Debra; and Yakusheva, Olga. Validation of the Registered Nurse Assessment of Readiness for Hospital Discharge Scale. Nursing Research, 67, 4: 305-313, 2018. Retrieved from Loyola eCommons, Nursing: School of Nursing Faculty Publications and Other Works, http://dx.doi.org/10.1097/NNR.0000000000000293

This Article is brought to you for free and open access by the Faculty Publications and Other Works by Department at Loyola eCommons. It has been accepted for inclusion in Nursing: School of Nursing Faculty Publications and Other Works by an authorized administrator of Loyola eCommons. For more information, please contact ecommons@luc.edu.

\section{(c) $($ †) $\ominus$}

This work is licensed under a Creative Commons Attribution-Noncommercial-No Derivative Works 3.0 License. (c) Wolters Kluwer Health, Inc. 2018 


\section{Validation of the Registered Nurse Assessment of Readiness for Hospital Discharge Scale} Abstract

Background: Statistical models for predicting readmissions have been published for high-risk patient populations, but typically focus on patient characteristics; nurse judgment is rarely considered in a formalized way to supplement prediction models.

Objectives: The purpose of this study was to determine psychometric properties of long and short forms of the RN-RHDS, including reliability, factor structure, and predictive validity. Methods: Data were aggregated from two studies conducted at 4 hospitals in the Midwestern United States. The RN-RHDS was completed within 4 hours before hospital discharge by the discharging nurse. Data on readmissions and ED visits within 30 days were extracted from electronic medical records.

Results: The RN-RHDS, both long and short forms, demonstrate acceptable reliability (Cronbach's alpha .90 and .73, respectively). Confirmatory factor analysis demonstrated less than adequate fit with the same 4-factor structure observed in the patient version. Exploratory factor analysis identified 3 factors, explaining $60.2 \%$ of the variance. When nurses rate patients as less ready to go home ( $<7$ out of 10$)$, patients are 6.4 to 9.3 times more likely to return to the hospital within 30 days, in adjusted models.

Discussion: The RN-RHDS, long and short forms, can be used to identify medical-surgical patients at risk for potential unplanned return to hospital within 30 days, allowing nurses to use their clinical judgment to implement interventions prior to discharge. Use of the RN-RHDS could enhance current readmission risk prediction models.

Keywords: discharge readiness, nurse assessment, readmissions 
Readiness for hospital discharge is a nurse-sensitive outcome of hospital care and an indicator of risk for adverse post-discharge outcomes that can lead to rehospitalization (Weiss et al., 2011). Acute care registered nurses are responsible for the process of preparing of patients for discharge (Nosbusch, Weiss, \& Bobay, 2010; Weiss et al., 2015). Typically, nurses assess discharge readiness informally within the context of preparing patients for discharge but there is not yet an evidence-based method or instrument in routine use to assist clinical nurses in a formal assessment of a patient's readiness for discharge. The purpose of this study is to validate an instrument for nurse (RN) assessment of readiness for hospital discharge.

Improvement in discharge transition care processes to achieve reduction in readmissions has become a priority for many hospitals in response to implementation of discharge quality metrics, such as HCAHPS (Hospital Consumer Assessment of Healthcare Providers and Systems) (AHRQ, 2017) and financial penalties for readmissions associated with the Affordable Care Act Hospital Readmissions Reduction Program (Mcllvennan, Eapen, \& Allen, 2015). Available readmission risk assessment tools are based on retrospective analyses of patient demographics and condition-specific parameters (Kansagara et al., 2011); none incorporate a systematic assessment of readiness for discharge by the discharging nurse prior to discharge.

The Readiness for Hospital Discharge Scale (PT-RHDS) was developed and tested in three patient populations (adult medical-surgical, postpartum mothers, parents of hospitalized children) to measure patient perception of discharge readiness at the time of discharge from acute care hospitalization (Weiss \& Piacentine, 2006; Weiss et al., 2007, 2008, 2009). Psychometric testing of the 21-item PT-RHDS supported reliability and validity of the scale when used with these patient populations (Weiss \& Piacentine, 2006; Weiss et al., 2011). Cronbach's alpha reliability estimates ranged from .83 to .93 for the total scale and .65 to .93 for the subscales. 
Confirmatory factor analysis validated the a priori theoretical structure of the scale (Weiss \& Piacentine, 2006; Weiss et al., 2011). Predictive validity testing with adult medical-surgical patients indicated that when patients assess that they are not ready for discharge, they are more likely to experience difficulties during the post-discharge period and have unplanned Emergency Department (ED) visits or readmissions within 30 days after discharge (Weiss et al., 2007; Weiss et al., 2011).

The items of the PT-RHDS were reworded to reflect the nurse as assessor to form a nurse assessment tool (RN-RHDS). In subsequent testing with a small sample of 162 nurse-patient pairs, the RN-RHDS but not the patient form (PT-RHDS) was found to be associated with readmission (Weiss et al., 2010). Scores on a short form of the RN-RHDS/SF (a parallel form of an 8-item PT- RHDS short form (PT-RHDS/SF) was also predictive of readmission in a sample of 254 nurse-patient pairs (Weiss et al., 2014).

While predictive associations of the RN-RHDS long form and short forms have been evident in previous studies (Weiss et al., 2010, 2014), construct validity has yet to be evaluated. Items of the RHDS scales were originally developed from content derived from literature sources and input from clinical nurse experts, with content validation by patients. The underlying factor structure was identified through factor analysis of patient-reported data on the PT-RHDS form (Weiss \& Piacentine, 2006). However, nurses may organize their thinking about discharge readiness differently than patients, producing a different factor structure for the nurse version of the scale. The aims of this secondary analysis are to evaluate the psychometric properties of the long form and short forms of the RN-RHDS, including reliability, factor structure, and predictive validity, determine concordance between parallel patient self-report and nurse assessment versions of the RHDS, and examine the utility of the RN-RHDS and RN-RHDS-SF as indicators 
of risk for return to the hospital for readmission or ED visits following discharge. Refinement of the RN-RHDS and validation of a short form based on analysis of the scale structure will provide a structured tool for clinical measurement by nurses of patients' readiness for discharge. The availability of a valid tool of a length suitable for use in practice settings and for integration into electronic health records will contribute to improved discharge transition efforts and readmission reduction.

\section{Methods}

\section{Study Design}

The study is situated within a conceptualization of hospital discharge as a transitional process, derived from Transitions Theory (Meleis et al., 2000) and Donabedian's (1966) Quality Model, that begins during hospital discharge preparation, has a transition point on the day of discharge, and is followed by a post-discharge period. Readiness for discharge is an outcome measure of the discharge preparation phase and an indicator of potential risks for coping difficulties and return to hospital during the post-discharge period (Weiss et al., 2015)

The design of this psychometric analysis of the RN-RHDS, a measure for nurse assessment of discharge readiness on the day of hospital discharge, included five sequential steps to: (1) test the adequacy of the a priori factor structure of the RN-RHDS, that was derived from the factor structure of the patient form of the RHDS, using confirmatory factor analysis; (2) explore possible alternative factor structures that may be unique to the RN-RHDS, using exploratory factor analysis; (3) evaluate the factor structure of a short form of the RN-RHDS (RN-RHDS/SF); (4) estimate the reliability of the RN-RHDS and RN-RHDS/SF; (5) determine the concordance between RHDS assessments by the nurse using RN-RHDS and patient self- 
report using the PT-RHDS; (5) determine the predictive validity of the RN-RHDS and RNRHDS/SF for return to hospital within 30 days post-discharge.

\section{Sample and Setting}

The sampling target for this study was 300 RN-RHDS assessments for an adequate sample for factor analysis (Comrey \& Lee, 1992). Participating nurses and patients were recruited from 16 medical-surgical nursing units in four hospitals within a health system in the Midwestern United States from April to August 2008 for the sample 1 and from the 6 of the same medical-surgical units in one of the hospitals from August 2012 to September 2013 for sample 2. Data were collected from patients and their discharging nurses on the day of discharge for both studies and by electronic data extraction of patient characteristics and post-utilization data from hospital information systems.

A total of 316 matched RN-patient pairs were included in this sample; 162 matched pairs were from a prior study (sample 1) (Weiss et al., 2010) and an additional 154 matched pairs (sample 2) were collected at a later date to achieve the sampling target. Both studies were approved by university and hospital Institutional Review Boards who approved a patient consent form for patients and an informational statement as the consent format for the nurse survey forms used for the study. Sample inclusion criteria were English or Spanish language patients at least 18 years old discharged to home without home hospice services. Nurses were approached for voluntary participation if their patient being discharged had agreed to participate. There was a total of 132 unique nurses who participated in sample 1 and 66 unique nurses who participated in sample 2 .

\section{Instruments and Measures}


The RN-RHDS was developed as a parallel measure of the PT-RHDS (Weiss \& Piacentine, 2006) to record nurse assessment of hospitalized patients' readiness for discharge by modifying the wording of items from patient-focused to nurse-focused questions. An example is "How well will you [patient form] / your patient [nurse form] be able to handle the demands of life at home?" For both the RN-RHDS and the PT-RHDS, there are 4 subscales: (1) Personal status; (2) Knowledge; (3) Coping ability; and (4) Expected Support. Personal status refers to how the patient feels physically and emotionally on the day of discharge and includes items related to how much pain or discomfort they are having, and their strength and energy levels. Knowledge relates to specific information the patient will need to self-manage personal and medical care needs, including possible complications, restrictions, and plan for follow-up. Coping ability refers to the patient's perception of ability to handle self-care, perform any treatments, or deal with demands of life at home. The Expected Support subscale measures whether the patient will have help with medical care or household activities after discharge (Weiss \& Piacentine, 2006). The RN-RHDS consists of a total of 21 items measured on a 0-10 Likert scale with higher scores indicating greater readiness. Scores are calculated and reported as mean of item scores, with a range of $0-10$. The RN-RHDS is administered on the day of discharge.

The 8-item RN-RHDS/SF is a parallel form of the PT-RHDS/SF which uses the 2 items from each subscale with the highest item-subscale correlations (Weiss et al., 2014). Initial testing of the RN-RHDS in 162 adult medical-surgical patients (sample 1) produced a Cronbach's alpha reliability estimate of .90 for the long form (Weiss et al., 2010) and .83 for the short form version (Weiss et al., 2014).

The outcome variables, readmissions and non-admitted ED visits within 30 days postdischarge, were extracted from electronic information systems through queries across the 4 study 
hospitals within the same health system. Readmissions and ED visit occurrences for any reason were counted if they occurred in any of the study hospitals. Readmissions not specifically related to the reason for the acute care hospitalization may reflect a general post-discharge syndrome characterized by the sequelae of hospitalization including physiologic and psychologic stresses of hospitalization that result in physiologic impairments and depletion of reserves needed for recovery and defense against adverse events (Krumholz, 2013). Due to few ED visits without concurrent readmission, the readmissions and ED visits were recoded into a single dichotomous variable, with $0=$ no return to hospital, and $1=$ one or more returns to hospital via ED visit or readmission.

Nurse and patient descriptive data were collected to compare first and second sample characteristics. $\mathrm{RN}$ descriptive data included years of experience as an $\mathrm{RN}$ and level of education, which were self-reported by the nurse at the end of the RN-RHDS form. Patient descriptive data included age in years, sex, ethnicity (Hispanic), lives alone, socioeconomic status (Hollingshead 4-Factor Index of Social Status, 1975), and diagnosis categorized as Major Diagnostic Category (MDC, www.cms.gov).

\section{Procedures}

Approval for this study was obtained from the health system and university Institutional Review Boards. Trained research assistants approached the nurses and patients for participation and obtained informed consent. Nurses completed the RN-RHDS within 4 hours before the patient's discharge from the acute care hospital.

\section{Data Analysis}

Descriptive statistics were calculated for RN and patient characteristics. Samples 1 and 2 were compared for similarity. T-tests were used for nurse and patient characteristics which were continuous measures; chi-square tests were used for categorical variables. Confirmatory factor 
analysis of the RN-RHDS was used to evaluate the a priori structure derived from the PT-RHDS, and subsequently exploratory factor analysis to identify alternative factor solutions. Concordance between the RN-RHDS and PT-RHDS was calculated using correlation and cross-tabulation. Predictive validity was evaluated with logistic regression models using RN-RHDS as the predictor variable and return to hospital within 30 days as the outcome variable, using age, sex, ethnicity, and lives alone, with MDC and unit fixed effects. Similar procedures were used for analysis of a short form version of the RHDS (SPSS, version 24, Chicago, IL).

\section{Results}

Table 1 summarized the sample demographics. In comparing samples 1 and 2 which were from different years but the same settings, sample 2 patients were significantly older (58.7 vs. $62.4, \mathrm{p}=.00$ ), more likely to live alone (22.8 vs. $26.1, \mathrm{p}=.02)$, more likely to be Hispanic (9.9 vs. $20.8, \mathrm{p}=.00)$, and more likely to return to the hospital ( 16.7 vs. $18.7, \mathrm{p}=.05)$. The samples were combined for the analyses.

\section{RN-RHDS}

Mean item scores on the 21-item RN-RHDS ranged from 7.9 to 9.2 on a 10-point Likert scale. The total scale Cronbach's alpha was .90. Inter-item correlations ranged from .34 to .78 and there were no corrected item-total correlations less than .3, indicating that items were measuring related content domains.

Subscale characteristics were evaluated through Pearson R inter-item correlations and Cronbach's alpha coefficients. The average Pearson R inter-item correlations for each of the subscales are as follows: Personal Status subscale average was .48 (range .27 to .78 ); Knowledge subscale average was .60 (range .35 to .84 ); Coping Ability subscale average was .73 (range .69 to .76); and Expected Support subscale average was .61 (range .41 to .80). Cronbach's alpha was 
assessed for each of the subscales and ranged from .78 for the Personal Status subscale to .92 for the Knowledge subscale, indicating adequate reliability.

\section{Confirmatory factor analysis of long form RN-RHDS}

A confirmatory factor analysis (CFA) using AMOS 22 (Chicago, IL) was conducted to test the fit of the RN-RHDS data to the a priori structure of the PT-RHDS. The model was specified with four latent variables representing the 4 subscales linked to their respective observed variables (items). The latent variables were allowed to correlate. For identification purposes, the path of one measured variable for each latent variable was set to 1 (Arbuckle \& Wothke, 1999). Missing values were replaced with substitution of subject-specific mean from the related subscale if less than $20 \%$ missing values. In interpreting the model, we examined $\chi^{2}$, root mean square error of approximation (RMSEA), and overall fit index. Because the $\chi^{2}$ is influenced by the sample size, we also looked at the $\chi^{2}$ to degrees of freedom (DF) ratio, where it has been suggested that a ratio of less than either 2 to 1 or 3 to 1 indicates an acceptable fit (Arbuckle \& Wothke, 1999; Ullman, 1996), although others suggest that less than 5 to 1 ratio may represent an acceptable fit (Kline 2005). We considered the following general "guidelines" that an RMSEA less than .05 indicates a "good fit" and less than .08 indicates an "acceptable fit," (McDonald \& Ho, 2002), though Hu \& Bentler (1999) suggest .06 for a "good fit,". Goodness of fit indices (such as CFI) should generally be larger than .90 (Hu \& Bentler, 1999; McDonald \& Ho, 2002).

The model resulted in a $\chi^{2}(183)=805.37, p<.01, \chi^{2} / \mathrm{df}$ ratio $=4.40, \mathrm{CFI}=.87$, and RMSEA $=.10$ with a $90 \%$ confidence interval of .097 to .111 . All of the items had statistically significant parameters on the a priori designated factor, $p<.01$ (See Table 2), indicating that the items were situated onto the correct factors, with the exception of a single item (physical ability 
to care for self after discharge) which loaded on Factor 2 (Knowledge) rather than Factor 1 (Personal Status) (Weiss et al., 2006). Correlations between factors ranged from .11 to .85 (Table 2); Personal status correlated with Knowledge at $\mathrm{r}=.51$ and Coping Ability at $\mathrm{r}=.58$.

Knowledge and Coping Ability were highly correlated at $r=>.80$ and Expected Support had low correlations with all other factors of less than $r=.30$. Because the Chi Square to DF ratio, and the RMSEA were slightly higher than ideal, the analysis progressed to exploratory factor analysis to identify a possible alternative underlying structure.

\section{Exploratory factor analysis of long form RN-RHDS}

The 21 items of the RN-RHDS long form were subjected to Principal Axis Factoring with Varimax rotation (allowing correlation among factors [Tabachnick \& Fidell, 2001]) using SPSS version 22 (Chicago, IL). Cross-loading values below 3 were suppressed. Adequacy of the sample was supported by the Kaiser-Meyer-Olkin values of .92, exceeding the recommended value of .6 (Pallant, 2013) and Bartlett's Test of Sphericity was significant at .00, supporting the factorability of the RN-RHDS.

Factoring revealed the presence of 3 components with eigenvalues exceeding 1 , explaining $41.5 \%, 11.7 \%$, and $6.9 \%$ of the variance, respectively (Table 2). There were no cross-loadings once values below .3 were suppressed. The rotated solution demonstrated three components with strong loadings and explained a total of $60.2 \%$ of variance within the scale. Factor 1 included items from the Personal Status, Knowledge, and Coping Ability subscales in the a priori structure. Two items from the Personal Status subscale loaded together on a Factor 2 - these items, strength and energy, were highly correlated ( $r=.78)$ and Factor 2 was correlated with Factor 1 at $r=.52$. Factor 3 included all items from the a priori Expected Support subscale. 
The resulting structure captures the inter-relatedness of Personal Status, Knowledge, and Coping Ability, and Expected Support as a dimension with low correlation to other factors reflecting what also emerged in the prior CFA analysis.

\section{RN-RHDS Short Form (RN-RHDS/SF)}

Given the similarities in factor structure from CFA of RN-RHDS in this analysis and CFA of PTRHDS in a prior study (Weiss \& Piacentine, 2006), we moved forward with testing a short form of the RN-RHDS/SF. The same two items from each of the 4 original subscales used in the PTRHDS/SF were used for the 8-item RN-RHDS/SF (the 8 items are noted in Table 2). As with the original PT-RHDS, the 8 items used in the RN-RHDS/SF had high item to subscale correlations (ranging from $r=.73$ to .90 ) in the RN-RHDS long form analysis. The Pearson correlation between the RN-RHDS long form and the short form was .96 and the RN-RHDS/SF explained $95 \%$ of the variance in the longer form scores.

Mean scores on the RN-RHDS/SF ranged from 7.9 to 8.9 on the $0-10$ point Likert scale. Internal consistency was examined using Cronbach's alpha coefficient, which was .73. The mean short form inter-item correlations ranged from .32 to .70 . As there are fewer than 10 items on the RN-RHDS/SF, it is recommended that particular attention be paid to the mean inter-item correlation (Pallant, 2010). No corrected item-total correlations were below .43.

Principal Axis Factoring was conducted on the 8 items of the RN-RHDS short form using SPSS version 24 (Chicago, IL). Inspection of the correlation matrix showed 7 out of $8(87.5 \%)$ coefficients of .3 and above and none were in a negative direction. The Kaiser-Meyer-Olkin value was .79 and Bartlett's Test of Sphericity was $.00(\mathrm{p}=.000)$, supporting factorability of the short form. 
The rotated solution demonstrated 2 clear components, both with strong factor loading values, and eigenvalues greater than 1, explaining $41.4 \%$ and $16.0 \%$ of the variance, respectively. The scree plot revealed a clear break after 2 components. Personal Status, Knowledge, and Coping Ability questions loaded onto one factor and the two Expected Support questions loaded on the second factor. The two factors were correlated at .21, suggesting that they are measuring different dimensions of readiness.

\section{Concordance with PT-RHDS}

Correlation between $\mathrm{RN}$ and patient versions was $.11(\mathrm{p}=.06)$ for the long forms and $.12(\mathrm{p}$ $=.04$ ) for the short forms. Using a previously established cut-off score for low readiness of less than 7 (Weiss et al., 2014), concordance between nurse and patient scores was $80.2 \%(235 / 293)$ agreement on being ready $(\geq 7), 2.0 \%(6 / 293)$ agreement on low readiness $(<7)$, and $17.7 \%$ (52/293) disagreement between nurse and patient. These findings indicate that, while most patients are ready for discharge as measured by parallel nurse assessment and patient self-report, in nearly one-fifth of cases, nurses and patients disagree on readiness for discharge.

\section{Positive Predictive Validity}

Positive predictive validity was estimated for both the long and short versions of the RNRHDS using return to hospital (occurrence of readmission and/or ED visit) within 30 days postdischarge as the outcome variable. All models included controls for patient characteristics (sex, age, socioeconomic status, ethnicity, lives alone, and socioeconomic status) which were associated with variation in readmission rates in previous studies (Kansagara et al., 2011; Weiss et al., 2010, 2011, 2014), with fixed effects for discharge unit, and clustering for nurses. The models were first estimated using the mean item scores for the long and short forms. Then the 
mean items scores on both forms were dichotomized using less than 7 as a cut-off score for low discharge readiness, based on prior analysis by the research team (Weiss et al., 2014). The models for mean and dichotomized scores were run unadjusted with no patient characteristics and adjusted with patient characteristics added. Results are presented in Table 3. Overall, the RN-RHDS long form was slightly more predictive than the short form (Odds Ratio [OR] $=3.34$ vs. $\mathrm{OR}=2.93$ in the unadjusted models, and $\mathrm{OR}=9.31$ vs. $\mathrm{OR}=6.36$ in the adjusted models, respectively). The results indicate that including the patient characteristics in the models improves predictive validity but unadjusted, as in the clinical practice situation, RN-RHDS was still associated with subsequent return to the hospital.

\section{Discussion}

The structural characteristics of the RN-RHDS 21-item long form are similar to what has been reported previously with the PT-RHDS (Weiss \& Piacentine, 2006) and remain consistent with the theoretical constructs in the literature. When some of the fit statistics of the 4-factor structure were slightly above recommended guidelines, the structure of the EFA revealed a coalescence of 3 of the 4 factors (Personal Status, Knowledge, Coping Ability) from the original scale. These factors are correlated indicating their reciprocal influence on each other. The EFA solution did not present an improvement over the original structure in terms of delineating the dimensions important to discharge readiness. In the analyses of both long and short forms, Expected Support is weakly related to the other subscale factors, and perhaps should be considered as a separate but related concept.

The reliability of the long form RN-RHDS is similar to previously reported PT-RHDS (Weiss et al., 2010). Short-form reliability is lower, but in the acceptable range, and is consistent 
with earlier estimates of reliability in the range of .75-.83 (Weiss et al., 2014); this may be expected as there were a smaller number of items which were specifically chosen to represent each of the 4 a priori domains; they were items with highest item-subscale correlations and not highest correlations with the scale as a whole. In keeping with the goal of creating a clinically useful nurse assessment tool, it is helpful for nurses to have similar questions on both the patient and $\mathrm{RN}$ versions of the forms for consistency and comparison. The short and long forms of the RN-RHDS demonstrate reliability, reasonable factoring results, and predictive validity suggesting that both forms are acceptable tools for assessing discharge readiness.

The results of this study demonstrate the value of nurse assessment in predicting postdischarge utilization. Discharge readiness is an outcome metric of hospital discharge process and a predictor of return to the hospital in the form of a readmission or ED visit. The results highlight the ability of nurses to anticipate patients at high risk for return to the hospital for readmission or ED visits who may need additional transitional care interventions to prevent return to hospital. The RN-RHDS was developed in response to clinical nurses' requests to provide a discharge assessment for their patients going home. Nurses felt that they were in the best position to know which patients were likely to be readmitted. Nurses are responsible for discharge preparation and may assess discharge readiness informally, but there has been no requisite or tool for formal assessment of discharge readiness on the day of discharge. The availability of a reliable and valid tool may promote standardized assessment of readiness for discharge which could be incorporated into electronic health records.

In this sample, correlations between nurse and patient assessments of discharge readiness are very low (less than $r=.15$ ) When we use a cut-off score for low readiness of less than 7 , established in a prior study (Weiss et al., 2014), concordance on readiness is high at $80 \%$ but 
agreement on low readiness is low, indicating that either the nurse or patient, but not both, recorded a low readiness score. Previous research has shown a persistent lack of agreement between RN and patient scores (Weiss et al., 2011, 2014). Explanations for this discrepancy may be a lack of communication between the nurse and patient or lack of awareness about the factors assessed in the tool. For example, nurses may be less aware of available support at home than patients themselves. In addition, nurses assess patients' discharge readiness relative to their experiences with previous patients; patients relate to their own unique perspective. needs after discharge. Another possible explanation is likely a result of nursing experience; having observed many similar patients, nurses may recognize implicit factors that patients do not that may contribute to return to hospital.

The long and short form RN-RHDS have different utilities for use in clinical practice. The RN-RHDS/SF is a screening tool that uses 2 items from each of the 4 subscales (Personal Status, Knowledge, Coping Ability, Expected Support) to evaluate discharge readiness. The RNRHDS long form offers the opportunity for a more complete assessment if indicated by low readiness scores on the short form. In both forms, low readiness scores are associated with substantially higher post-discharge return to hospital rates (3-9 times). The scales can potentially be used as one method for evaluating return to the hospital risk. Although there are several readmission risk-identification scoring tools, these current risk assessment models are based on retrospective large dataset analyses incorporating diagnostic, clinical condition, and demographic factors, but rarely indicators of overall health and function, severity of illness, or social determinants of health (Kansagara et al., 2011). Primary data collection at or near discharge is largely absent in risk assessment tools; in particular, these tools do not include direct assessments 
of patient condition and functional status at discharge, knowledge of self-care and perceived abilities, and the level of expected support. These factors are assessed in the RN-RHDS.

The value of the RN-RHDS tools may lie in augmenting existing readmission risk determination models by shifting to prospective assessment for identifying patients for unplanned return to hospital. Future studies are needed to evaluate the prospective use of the RN-RHDS in conjunction with typical risk identification by diagnosis and demographics characteristics associated with readmission. Knowing which patients are likely to return to hospital is of vital interest for hospitals as they work fine-tune efforts to identify patients at high risk for readmissions for which the hospital is no longer reimbursed.

In clinical practice, the RN-RHDS offers the discharging nurse a tool for screening patients for low readiness in anticipation of the discharge. This end-of-hospitalization screening could identify patients before discharge who need supplemental efforts to prepare for discharge and/or additional post-discharge transitional care services to mitigate the risks associated with low readiness. Implementation of the RN-RHDS as a standard nursing practice prior to discharge would offer a systematic approach to triggering initiation of targeted nurse actions in response to low discharge readiness assessments focused on improving the transition to home and reducing the risk of post-discharge problems that lead to return to the hospital.

Strengths of the study include that the sample was derived from multiple nursing units in multiple hospitals and represented a broad range of adult medical-surgical patients discharged from hospitals. The tools are applicable broadly as an outcome measure of discharge preparation (Weiss et al., 2015) and prospective identification of risk for readmission. A limitation of the study includes capture of return to hospital only to the four study hospitals involved, not to other 
hospitals outside of the healthcare system. Measuring same hospital occurrences canmiss up to $20 \%$ of readmissions, thereby underestimating the actual number of readmissions (Nasir et al. 2010). All-cause readmissions were counted; data were not available in the study datasets to exclude planned readmissions. We did not collect data about discharge preparation or readmission reduction programs on study units. We accounted for any differences between units by using unit fixed effects in regression models for predictive validity. The sample included 316 unique discharge events. A single nurse may be included several times in the database discharging several patients. We addressed this by including clustering for nurses in the analysis. There was no reliable method to measure nurse expertise, which may have affected the assessments. Another limitation was that the sample only included adult medical-surgical patients. Results for specific groups of adult patients, such as oncology or other diagnosisspecific categories may produce different results. The data in the two study samples was collected 6 and 10 years ago. Recognizing that the complexity of health care has increased and many hospitals have implemented discharge process improvement initiatives, the relevance of the nurse and patient measures could have changed. Research conducted with the instruments in the intervening period continues to support the relevance and utility. While the data are dated, the data set used represents the only data available to date with matched patient and nurse readiness assessments in short and long forms needed for parts of the psychometric analysis.

\section{Conclusion}

The RN-RHDS in long and short forms are reliable. The long form RN-RHDS has a similar factor structure to the patient version of the RHDS. Both forms demonstrate predictive validity for return to hospital (readmissions and ED visits within 30 days of discharge). The study demonstrates the value of nurse assessments of discharge readiness in contributing to 
efforts to improve the transition to home and decrease unplanned return to hospital. If assessment of discharge readiness becomes a standard nursing practice, nurses will be able to more effectively evaluate their valuable contribution in preparing the patient for discharge to improve post-discharge outcomes. 


\section{References}

Agency for Healthcare Research and Quality. (2017). CAHPS Adult Hospital Survey. Retrieved from http://www.ahrq.gov/cahps/surveys-guidance/hospital/about/adult hp survey.htm.

Arbuckle, J. L. \& Wothke, W. (1999). AMOS 4.0 user's guide. Chicago IL: Small Waters Corp.

Centers for Medicare and Medicaid Services. (2017). FY 2017 Final Rule and Correction Notice Tables. Retrieved from https://www.cms.gov/Medicare/Medicare-Fee-for-ServicePayment/AcuteInpatientPPS/FY2017-IPPS-Final-Rule-Home-Page-Items/FY2017-IPPSFinal-Rule-Tables.html

Comrey, A. L. \& Lee, H. B. (1992). Interpretation and application of factor analytic results.

In Comrey, A. L. \& Lee, H. B. (Eds.), A first course in factor analysis (pp. 240-

263). Hillsdale, NJ: Lawrence Erlbaum.

Donabedian, A. (1966). Evaluating the quality of medical care. Milbank Memorial Fund Quarterly, 44(3, suppl), 166-206. PMID: 16279964

Hollingshead, A. (1975). Four factor index of social status. New Haven, CT: Yale University.

Hu, L., \& Bentler, P. M. (1999). Cutoff criteria for fit indexes in covariance structure analysis: conventional criteria versus new alternatives. Structural Equation Modeling, 6, 1-55. DOI: $10.1080 / 10705519909540118$.

Kansagara, D., Englander,H., Salanitro, A., ..... and Kripalani, S. (2011). Risk prediction models for hospital readmission: a systematic review. Journal of the American Medical Association, 306(15), 1688-98. DOI: 10.1001/jama.2011.1515

Kline, R. B. (2005). Principles and practice of structural equation modelling, $2^{\text {nd }}$ edition. New York: Guilford.McDonald, R., \& Ho, M. R. (2002). Principles and practices in reporting structural equation analyses. Psychological Methods, 7, 64-82. DOI: 10.1037//1082$989 x .7 .1 .64$

Krumholz, H.H. (2013). Post-hospital syndrome - an acquired, transient condition of generalized risk. New England Journal of Medicine, 386(2), 100-102. DOI: 10.1056/ NEJMp1212324 
McDonald, R.P. \& Ho, M.-H. (2002). Principles and practice in reporting structural equation analyses. Psychological Methods, 7(1), 64-82. Doi.apa.org

McIlvennan, C. K., Eapen, Z. J., \& Allen, L. A. (2015). Hospital Readmissions Reduction Program. Circulation, 131(20), 1796-1803. http://doi.org/10.1161/CIRCULATIONAHA.114.010270

Meleis, A.I., Sawyer, L.M., Im, E., Messias, K.H., \& Schumacher, K. (2000). Experiencing transitions: an emerging middle-range theory. Advances in Nursing Science, 23(1), 12-28. PMID: 10970036

Nasir, K., Lin, Z., Bueno, H., .... \& Krumholz, H.M. (2010). Is same-hospital readmission rate a good surrogate for all-hospital readmission rate? Medical Care, 48 (5), 477-81. DOI: 10.1097/MLR.0b013e3181d5fb24

Nosbusch, J.M., Weiss, M.E., \& Bobay, K.L. (2010). Challenges confronting the acute care staff nurse in discharge planning: an integrated review of the literature. Journal of Clinical Nursing, 20 (5-6), 754-774. DOI: 10.1111/j.1365-2702.2010.03257.x

Pallant, J. (2013). SPSS survival manual: a step by step guide to data analysis using SPSS, $5^{\text {th }}$ ed., Berkshire, England: McGraw-Hill.

Tabachnick, B.G. \& Fidell, L.S. (2001). Using multivariate statistics (4 ${ }^{\text {th }}$ ed.). Boston, MA: Allyn \& Bacon.

Ullman, J. B. (1996). Structural equation modeling. In B. Tabachnick \& L Fidell, Using multivariate statistics, $3^{\text {rd }}$ ed. (pp. 709-812). New York: Harper Collins.

Weiss, M.E, Bobay, K.L., Bahr, S.J., Costa, L., Hughes, R., \& Holland, D.E. (2015).

A conceptual model for hospital discharge preparation. Journal of Nursing Administration, 45(12), 606-614. DOI: 10.1097/NNA.0000000000000273

Weiss, M.E., Costa, L.L., Yakusheva, O., \& Bobay, K.L. (2014). Validation of patient and nurse short forms of the Readiness for Hospital Discharge Scale and their relationship to return to the hospital. Health Services Research, 49(1), 304-317. DOI: 10.1111/1475-6733.12092

Weiss, M. E. \& Lokken,L. (2009). Predictors and outcomes of postpartum mothers' perceptions of readiness for discharge after birth. Journal of Obstetric, Gynecologic, \& Neonatal Nursing, 38, 406-17. DOI: 10.1111/j.1552-6909.2009.01040.x.

Weiss, M., Johnson, N., Malin, S., Jerofke, T., Lang, C., \& Sherburne, E.. (2008). Readiness for discharge in parents of hospitalized children. Journal of Pediatric Nursing, 23(4), 282- 
95. DOI: $10.1016 /$ j.pedn.2007.10.005

Weiss, M.E. \& Piacentine, L.B. (2006). Psychometric properties of the Readiness for Hospital Discharge Scale, Journal of Nursing Measurement, 14 (3), 163-180. DOI: http://dx.doi.org/10.1891/jnm-v14i3a002

Weiss, M. E., Piacentine, L.B., Lokken, L., ....., \& Vega-Stromberg, T. (2007). Perceived readiness for hospital discharge in adult medical-surgical patients. Clinical Nurse Specialist, 21(1), 31-42. PMID: 17213738

Weiss, M.E., Yakusheva, O., \& Bobay, K.L. (2010). Nurse and patient perceptions of discharge readiness in relation to postdischarge utilization. Medical Care, 48(5), 482-486. DOI: 10.1097/MLR.0b013e3181d5feae

Weiss ME, Yakusheva O, Bobay KL. (2011). Quality and cost analysis of nurse staffing, discharge preparation, and postdischarge utilization. Health Services Research, 46(5), 14731494. DOI: $10.1111 / j .1475-6773.2011 .01267 . x$ 